\title{
Checagem corporal, atitude alimentar inadequada e insatisfação com a imagem corporal de jovens universitários
}

\author{
Body-checking, inappropriate eating attitudes and body \\ image dissatisfaction among young students
}

Pedro Henrique Berbert de Carvalho', Juliana Fernandes Filgueiras', Clara Mockdece Neves', Fernanda Dias Coelho², Maria Elisa Caputo Ferreira'

\section{RESUMO}

Objetivo: $O$ objetivo do estudo foi avaliar a checagem corporal, a atitude alimentar inadequada e a insatisfação corporal em universitários de ambos os sexos. Métodos: Participaram 587 indivíduos (311 homens). Foram avaliados os comportamentos de checagem (mulheres - Body Checking Questionnaire; homens - Male Body Checking Questionnaire), as atitudes alimentares inadequadas (Eating Attitudes Test-26) e a insatisfação corporal (Body Shape Questionnaire). Massa corporal e estatura foram autorreferidas para o cálculo do índice de massa corporal (IMC). Foi realizada estatística descritiva, inferencial e teste qui-quadrado (teste exato de Fisher) utilizando o software Statistic 8.0 e adotado nível de significância de 5\%. Resultados: A checagem corporal está associada às atitudes alimentares inadequadas e à insatisfação corporal, independentemente do sexo do indivíduo. Foram encontradas diferenças entre o sexo feminino e masculino para as variáveis avaliadas, sendo maior a frequência entre

\section{Palavras-chave}

Atitude alimentar, imagem corporal, sexo. as mulheres. Conclusão: Concluiu-se que a checagem corporal é um comportamento presente e de alta frequência em universitários com risco para o transtorno alimentar e também naqueles insatisfeitos com sua imagem corporal.

\section{ABSTRACT}

Objective: The aim of this study was to evaluate the body-checking, inappropriate eating attitudes and body dissatisfaction in undergraduate students from both sexes. Methods: Five hundred eighty-seven undergraduate students (311 men) were surveyed. The body-checking behaviours (women - Body Checking Questionnaire; men - Male Body Checking Questionnaire), inappropriate eating attitudes (Eating Attitudes Test-26) and body dissatisfaction (Body Shape Questionnaire) were evaluated. Body mass and height were self-referred to calculate the body mass index (BMI). Descriptive, inferential and qui-square test (Fisher exact test) statistics were performed using the Statistic 8.0 software and adopting significant level of 5\%. Results: Body-checking behaviours are associated with inappropriate eating attitudes and body dissatisfaction in both sexes. Women and men showed differences between the varia-

1 Universidade Federal de Juiz de Fora (UFJF), Faculdade de Psicologia, Laboratório de Estudos do Corpo (Labesc), Programa de Pós- Graduação em Psicologia.

2 UFJF, Faculdade de Educação Física, Labesc, Programa de Pós-Graduação em Educação Física.

Endereço para correspondência: Pedro Henrique Berbert de Carvalho

Faculdade de Educação Física e Desportos, Universidade Federal de Juiz de Fora Rua José Lourenço Kelmer, s/n, São Pedro - 36036-900 - Juiz de Fora, MG, Brasil

E-mail: pedro.berbert@gmail.com 


\section{Keywords}

Eating attitudes, body

image, sex. bles evaluated, being higher in women. Conclusion: Body-checking is a present and high frequency behaviour in undergraduate students with risk for eating disorders and also with those dissatisfied with their body image.

\section{INTRODUÇÃO}

A insatisfação com a imagem corporal, entendida como um sentimento negativo que o indivíduo tem em relação ao seu peso e à sua forma corporal, é um aspecto importante para o diagnóstico de alguns transtornos alimentares, como a anorexia e a bulimia nervosas'. A insatisfação com o corpo está associada a fatores prejudiciais à saúde, como baixa autoestima, depressão, estados de ansiedade social e, principalmente, a atitudes inadequadas de controle do peso, como o abuso de laxantes, uso de substâncias anorexígenas e esteroides anabólicos, e comportamentos alimentares inadequados².

Entende-se por comportamento alimentar todas as formas de convívio com o alimento ${ }^{3}$, sendo a insatisfação corporal identificada como o principal fator de risco para o desenvolvimento do comer transtornado 4 . Segundo Alvarenga et al. 5 , o comportamento alimentar inadequado é frequente em universitários que apresentam relação conturbada com o alimento e com o corpo, podendo ter consequências físicas e psíquicas.

Indivíduos insatisfeitos com sua imagem corporal tendem, ainda, a adotar comportamentos de checagem corporal acompanhados por períodos de restrição alimentar'. Rituais de pesagens, medidas e comparações de seu corpo com o de outros indivíduos repetem-se compulsivamente, possuindo papel mantenedor da imagem corporal negativa pela atenção seletiva e constante à forma e ao peso corporal, e serve como parâmetro de avaliação do indivíduo no julgamento de seu sucesso ou sua falha no controle do peso?.

A checagem corporal não apresenta validade diagnóstica para os transtornos alimentares, no entanto está associada ao seu aspecto psicopatológico central, à insatisfação corporal ${ }^{8}$, e tem, portanto, importância clínica. Ações no sentido de diminuição desse comportamento em pacientes com transtornos alimentares têm sido frequentes ${ }^{9,10}$ e são apontadas como alternativa de intervenção para o distúrbio de imagem corporal ${ }^{8}$.

O comportamento de checagem corporal também está presente na população não clínica ${ }^{11,12}$. Os resultados apontam para uma influência direta da checagem corporal na atenção seletiva do indivíduo para o seu corpo, com aumento da insatisfação sobre ele ${ }^{11}$.

O ingresso na vida universitária provoca alterações no comportamento alimentarr', e a exposição ao novo meio social pode trazer como consequência aumento da insatisfação corporal. Estudos têm sido realizados no sentido de avaliar a insatisfação com o corpo e também as atitudes alimen- tares inadequadas em universitários ${ }^{14-16}$, porém a associação da checagem corporal com essas variáveis ainda é pouco explorada pelos pesquisadores.

Dessa forma, o presente estudo objetivou avaliar a checagem corporal, a atitude alimentar inadequada e a insatisfação corporal em universitários de ambos os sexos.

\section{MÉTODOS}

\section{Amostra}

Este estudo do tipo transversal foi desenvolvido com 587 universitários (276 mulheres e 311 homens), de diversos cursos de graduação (Ciências da Computação, Direito, Engenharia Civil, Engenharia Elétrica, Filosofia, Fisioterapia, Matemática, Medicina e Psicologia), da Universidade Federal de Juiz de Fora (UFJF). O cálculo da amostra foi baseado nas recomendações de Thomas et al. ${ }^{17}$, considerando um grau de confiança igual a 95\%, erro máximo de estimativa de 5\% e prevalência do fenômeno desconhecida, inferido, portanto, em um valor de 50\%. O valor obtido, já corrigido para amostras finitas, foi de 378 indivíduos. Foram acrescidos 20\%, para efeito de perda de amostra, obtendo-se um valor final igual a 454 indivíduos.

O critério de inclusão era estar regularmente matriculado em qualquer período de um dos cursos de graduação da UFJF, ter idade superior a 18 anos e aceitar participar voluntariamente da pesquisa.

Este estudo foi aprovado pelo Comitê de Ética em Pesquisa em Seres Humanos da UFJF - protocolo no 2193.253.2010 (parecer no 276/2010). Todos os indivíduos foram informados sobre os objetivos e riscos da pesquisa e concordaram em participar dela por meio da assinatura do Termo de Consentimento Livre e Esclarecido (TCLE).

\section{Instrumentos}

\section{Comportamento de checagem corporal}

Para avaliar os comportamentos de checagem do corpo no sexo feminino, foi utilizado o Body Checking Questionnaire $(\mathrm{BCQ})^{18,19}$, questionário de autorrelato em escala na forma $\mathrm{L} i$ kert de cinco pontos (Nunca $=1$ a Muito frequentemente = 5). O instrumento original é formado por três fatores e obteve consistência interna satisfatória (a-Cronbach variando de $0,83$ a 0,92$)^{18}$. A versão brasileira obteve adequada estrutura fatorial confirmada pelas medidas de ajuste e adequada consistência interna, possui 12 itens e é subdividida em quatro 
fatores: checagem pela observação do corpo, checagem por meio de medições de partes do corpo, checagem com comparações entre o corpo do sujeito e os corpos de outros e busca por informações perceptivas ${ }^{19}$. O escore varia de 12 a 60 pontos. Quanto maior o escore, mais frequente é o comportamento de checagem corporal ${ }^{19}$. Para a amostra em questão, a consistência interna obtida foi de 0,85.

A avaliação da checagem corporal dos indivíduos do sexo masculino foi realizada pelo Male Body Checking Questionnaire $(\mathrm{MBCQ})^{20-22}$. O MBCQ é um instrumento de autorrelato com 19 itens, respondidos em escala na forma Likert de cinco pontos (Nunca $=1$ a Muito frequentemente $=5$ ), em que os indivíduos, no atual momento, devem avaliar a frequência em que adotam determinados comportamentos. O escore varia de 19 a 95 pontos. Quanto maior a pontuação, mais frequentes são os comportamentos relacionados à checagem corporal22. O MBCQ foi desenvolvido como alternativa ao $B C Q$, pela possibilidade de avaliação de comportamentos de indivíduos do sexo masculino ${ }^{20}$. O estudo original demonstrou a existência de quatro fatores com adequada consistência interna de 0,94 (a-Cronbach variando de 0,72 a $0,86)^{20}$. A versão brasileira comprovou a existência de quatro fatores (comparação com outras pessoas; uso de superfície reflexiva; checagem por "pinçamento" - gordura; e checagem por avaliação externa) e obteve adequada consistência interna ( $a$-Cronbach $=0,93)^{21}$. Na presente amostra a consistência interna correspondeu a 0,94.

\section{Insatisfação com a imagem corporal}

Para avaliar a insatisfação e as preocupações com a forma do corpo, foi utilizado o Body Shape Questionnaire (BSQ) $)^{23,24} \mathrm{em}$ sua versão validada para uma população de universitários brasileiros de ambos os sexos ${ }^{24}$. O questionário é composto por 34 questões, em escala na forma Likert de seis pontos (Nunca $=1$ a Sempre $=6$ ), em que o avaliado aponta com que frequência, nas últimas quatro semanas, vivenciou os eventos propostos pelas alternativas. A versão brasileira obteve adequada estrutura fatorial, dividida em quatro fatores (autopercepção da forma corporal; percepção comparativa da imagem corporal; atitudes em relação à alteração da imagem corporal; e alterações severas na percepção corporal) e adequada consistência interna ${ }^{24}$. A classificação dos resultados do BSQ é dividida em quatro níveis de insatisfação corporal: abaixo de 110 pontos, livre de insatisfação; entre 111 e 138, insatisfação leve; entre 139 e 167, insatisfação moderada; e pontuação igual ou acima de 168 indica grave insatisfação corporal ${ }^{23,24}$. Para a amostra total, foi obtido alfa de 0,95 (0,95 para as mulheres e 0,92 para os homens).

\section{Atitudes alimentares inadequadas}

A avaliação das atitudes alimentares inadequadas se deu por meio do Eating Attitudes Test (EAT-26) ${ }^{25,26}$, composto por 26 questões de autorrelato abordando atitudes alimentares.
As respostas variam de sempre (3 pontos) a nunca (0 ponto), exceto a questão 25 , que apresenta pontuação inversa. $\mathrm{O}$ escore igual ou superior a 21 pontos é indicador de possíveis comportamentos de risco para o transtorno alimentar (EAT-26 positivo), além da presença de padrões alimentares anormais, não revelando, contudo, a possível psicopatologia subjacente ao comportamento manifesto ${ }^{26}$. A versão brasileira apresenta satisfatória consistência interna ${ }^{26}$. Para a amostra total, foi obtido alfa de 0,88 (0,87 para as mulheres e 0,88 para os homens).

\section{Avaliação do estado nutricional}

Para a avaliação do estado nutricional, foi utilizado o cálculo do índice de massa corporal (IMC), por meio dos dados de massa corporal e estatura autorreferidos pelos participantes. Estudos têm demonstrado que os indivíduos são capazes de relatar de forma acurada seu peso e estatura corporal|27,28. A classificação foi realizada a partir dos pontos de corte do IMC, estabelecidos pela Organização Mundial da Saúde ${ }^{29}-\mathrm{IMC}<$ $18,5 \mathrm{~kg} / \mathrm{m}^{2}$ (baixo peso); IMC 18,5-24,9 kg/m² (eutrofia); IMC $25-29,9 \mathrm{~kg} / \mathrm{m}^{2}$ (sobrepeso); e IMC > 29,9 kg/m² (obesidade).

\section{Procedimentos}

Após a aprovação do projeto, os pesquisadores estabeleceram contato com professores para explicar todo o procedimento adotado e pedir autorização para intervenção durante o período inicial de sua aula. Foram selecionados os melhores dias para a coleta, que ocorreram entre os meses de agosto e setembro de 2011.

No dia de coleta, os pesquisadores realizaram uma explicação sobre os objetivos e procedimentos da pesquisa para os alunos e distribuíram o TCLE. Assim, aqueles que aceitaram participar voluntariamente da pesquisa, por meio da assinatura do termo, responderam aos questionários de avaliação.

\section{Análise estatística}

Todos os dados foram armazenados em um banco de dados e analisados por meio do software Statistic 8.0. Os indivíduos foram agrupados de acordo com a classificação de sua imagem corporal (satisfeitos - livre de insatisfação; e insatisfeitos - leve, moderada e grave insatisfação corporal) e da presença de sintomas relacionados aos transtornos alimentares (EAT-26 positivo e EAT-26 negativo).

Pela impossibilidade de classificação dos indivíduos quanto aos comportamentos de checagem corporal (não normatização dos escores do BCQ e $\mathrm{MBCQ}$ ), eles foram subdivididos em dois grupos (baixa e alta checagem). Para tanto, foram classificados como alta checagem aqueles que responderam a todos os itens dos questionários com os escores frequentemente ou muito frequentemente (escala na forma Likert 4 ou 5 pontos).

As variáveis foram descritas em medidas de tendência central (média) e de dispersão (desvio-padrão). As prevalên- 
cias de checagem corporal, atitudes alimentares inadequadas e de insatisfação corporal foram descritas em frequência absoluta e relativa. A estatística inferencial foi aplicada para relatar a consistência interna ( $a$-Cronbach) dos questionários.

Foi realizada comparação dos sexos quanto às variáveis utilizadas (checagem corporal, atitude alimentar inadequada e insatisfação corporal) e também entre os grupos de baixa e alta checagem corporal. Para tanto, utilizou-se o teste quiquadrado (teste exato de Fisher), com 95\% de intervalo de confiança. Para todas as análises foi considerado como significativo um $p<0,05$.

\section{RESULTADOS}

Participaram do estudo 587 universitários (311 homens) com idade média de 20,98 anos (DP = 3,65). Desses, 77,51\% ( $n=$ 455) eram eutróficos, $18,91 \%(n=111)$ estavam com sobrepeso e $3,58 \%(n=21)$, obesos.

A tabela 1 descreve os dados de checagem corporal, atitude alimentar inadequada e insatisfação com a imagem corporal, divididos por sexo.

Tabela 1. Dados descritivos da amostra, de acordo com os escores brutos obtidos em cada instrumento, divididos por sexo - Juiz de Fora (MG), 2012

\begin{tabular}{lcc}
\hline \multicolumn{1}{c}{ Variáveis } & $\begin{array}{c}\text { Feminino }(\boldsymbol{n}=276) \\
\mathbf{M}(\mathbf{D P})\end{array}$ & $\begin{array}{c}\text { Masculino }(\mathbf{n}=311) \\
\mathbf{M}(\mathbf{D P})\end{array}$ \\
\hline Checagem corporal $^{\sharp}$ & $24,45(8,03)$ & $34,03(13,10)$ \\
Atitude alimentar inadequada & $13,27(9,89)$ & $8,81(9,13)$ \\
Insatisfação com a imagem corporal & $79,09(30,71)$ & $57,40(19,59)$ \\
\hline${ }^{*}$ Checagem corporal de acordo com o instrumento adequado (sexo feminino - BCQ; sexo masculino - MBCQ). \\
$M=$ média; DP = desvio-padrão.
\end{tabular}

Foram identificadas diferenças estatisticamente significativas para a prevalência das variáveis avaliadas entre os sexos, sendo maior a frequência de checagem corporal, EAT-26 positivo e insatisfação corporal nas mulheres (Tabela 2).

Tabela 2. Comparação entre os sexos das variáveis checagem corporal, atitude alimentar inadequada e insatisfação com a imagem corporal - Juiz de Fora (MG), 2012

\begin{tabular}{lcccc}
\hline \multicolumn{1}{c}{ Variáveis } & Feminino $(\mathrm{n}=276)$ & Masculino $(\mathrm{n}=311)$ & df & p-valor \\
\hline Checagem corporal & & & & \\
Baixa & & & & \\
Alta & $242(91,30 \%)$ & $305(98,07 \%)$ & 13,81 & $0,002^{*}$ \\
Atitude alimentar inadequada & & $6(1,93 \%)$ & & \\
EAT-26 negativo & $214(77,54 \%)$ & $291(93,57 \%)$ & 31,28 & $0,001^{*}$ \\
EAT-26 positivo & $62(22,46 \%)$ & $20(6,43 \%)$ & & \\
Insatisfaçäo com a imagem corporal & & & & \\
Satisfeito & $228(82,60 \%)$ & $304(97,75 \%)$ & 39,47 & $0,001^{*}$ \\
Insatisfeito & $48(17,40 \%)$ & $7(2,25 \%)$ & & \\
\hline
\end{tabular}

"Checagem corporal de acordo com o instrumento adequado (sexo feminino - BCQ; sexo masculino - MBCQ).

* Teste qui-quadrado (teste exato de Fisher). Significativo para $p<0,05$.
A comparação entre os grupos com baixa e alta frequência de comportamentos de checagem corporal apresentou diferença estatisticamente significativa para as variáveis atitudes alimentares inadequadas e insatisfação com a imagem corporal, tanto para o sexo feminino (Tabela 3), quanto para o masculino (Tabela 4).

Tabela 3. Comparação entre os grupos de baixa e alta frequência de checagem corporal do sexo feminino quanto à atitude alimentar inadequada e à insatisfação com a imagem corporal Juiz de Fora (MG), 2012

\begin{tabular}{lcccc}
\hline \multirow{2}{*}{ Variáveis } & \multicolumn{2}{c}{ Checagem corporal } & df & $p$-valor \\
\cline { 2 - 3 } & \multicolumn{1}{c}{ Baixa } & Alta & & \\
\hline Atitude alimentar inadequada & & & & \\
EAT-26 negativo & $202(73,19 \%)$ & $12(4,34 \%)$ & 11,44 & $0,007^{*}$ \\
EAT-26 positivo & $50(18,12 \%)$ & $12(4,34 \%)$ & & \\
Insatisfação com a imagem corporal & & & \\
Satisfeito & $200(72,46 \%)$ & $28(10,15 \%)$ & 78,05 & $0,001^{*}$ \\
Insatisfeito & $14(5,07 \%)$ & $34(12,32 \%)$ & & \\
\hline
\end{tabular}

* Teste qui-quadrado (teste exato de Fisher). Significativo para $p<0,05$.

Tabela 4. Comparação entre os grupos de baixa e alta frequência de checagem corporal do sexo masculino quanto à atitude alimentar inadequada e à insatisfação com a imagem corporal Juiz de Fora (MG), 2012

\begin{tabular}{|c|c|c|c|c|}
\hline \multirow{2}{*}{ Variáveis } & \multicolumn{2}{|c|}{ Checagem corporal } & \multirow{2}{*}{$d f$} & \multirow{2}{*}{ p-valor } \\
\hline & Baixa & Alta & & \\
\hline \multicolumn{5}{|c|}{ Atitude alimentar inadequada } \\
\hline EAT-26 negativo & $283(91,00 \%)$ & $8(2,57 \%)$ & 18,93 & $0,001^{*}$ \\
\hline EAT-26 positivo & $17(5,46 \%)$ & $3(0,97 \%)$ & & \\
\hline \multicolumn{5}{|c|}{ Insatisfação com a imagem corporal } \\
\hline Satisfeito & $299(96,14 \%)$ & $5(1,61 \%)$ & 22,55 & $0,001^{*}$ \\
\hline Insatisfeito & $5(1,61 \%)$ & $2(0,64 \%)$ & & \\
\hline
\end{tabular}

\section{DISCUSSÃO}

Este estudo avaliou a frequência dos comportamentos de checagem corporal, as atitudes alimentares inadequadas e a insatisfação corporal de universitários de ambos os sexos.

É reconhecida na comunidade científica a associação da insatisfação com o corpo e as atitudes alimentares inadequadas. Os distúrbios de imagem corporal são aspectos importantes para o diagnóstico de diversos transtornos mentais como a anorexia e bulimia nervosas e também o transtorno dismórfico corporal' ${ }^{1}$. No entanto, ao menos no Brasil, poucos estudos avaliaram comportamentos adotados rotineiramente pela população, como a pesagem corporal, a comparação de seu corpo com o de outros indivíduos e a verificação do corpo em superfícies reflexivas (por exemplo: espelho), e em 
especial sua associação com as atitudes alimentares inadequadas e a insatisfação corporal.

Foi identificado reduzido número de indivíduos com alta frequência de checagem corporal, existindo diferenças entre os sexos feminino e masculino $(8,7 \%$ vs. $1,93 \% ; p=0,002)$. Alfano et al. ${ }^{12}$ sugerem o uso de instrumentos específicos para cada sexo (BCQ - mulheres; MBCQ - homens) por causa das idiossincrasias encontradas quanto à expectativa de corpo e rituais de autoavaliação dele. Esta pesquisa teve esse cuidado metodológico ao utilizar instrumentos específicos e identificou maior frequência de checagem entre as mulheres, o que reforça o fato de estas apresentarem comportamento mais frequente de autoavaliação do corpo, geralmente acompanhado de insatisfação corporal, quando comparadas aos homens ${ }^{12}$.

Vale ressaltar que, para a classificação de alta frequência de checagem corporal, foram adotados os pontos de corte da escala Likert (4 e 5 pontos). Esses valores correspondem a "frequentemente" e "muito frequentemente", respectivamente, o que confere a esses indivíduos um padrão acentuado de autoavaliação corporal. Segundo Fairburn et al.? indivíduos que se engajam em um alto padrão de checagem corporal tendem a aumentar sua insatisfação com o corpo e a adotar estratégias inadequadas para o controle do peso e/ ou modificação corporal. Estes são considerados fatores de risco para o desenvolvimento de distúrbios de imagem corporal e transtornos alimentares, portanto a checagem corporal deve ser encarada como comportamento importante no acompanhamento do indivíduo com e sem transtorno alimentar.

Em relação às atitudes alimentares inadequadas, foi identificada alta frequência de mulheres com comportamentos de risco para o desenvolvimento de transtornos alimentares, diferindo estatisticamente dos homens (22,46\% vs. 6,43\%; $p$ $=0,001)$. Alvarenga et al..$^{5}$ encontraram uma frequência que variou de $23,7 \%$ a 30,1\% em jovens universitárias das cinco regiões do Brasil, utilizando o mesmo instrumento avaliativo (EAT-26), com ponto de corte igual ou superior a 21 pontos. Já Nunes et al.30, em estudo de base populacional realizado em Porto Alegre, utilizando ponto de corte superior a 21 pontos, identificaram uma frequência de 16,5\%. Cumpre ressaltar que a atitude alimentar inadequada é fator de risco para o desenvolvimento de transtornos alimentares, que, juntamente com a insatisfação com o corpo, pode ter importantes consequências físicas e psíquicas para a vida do indivíduo ${ }^{5}$.

Segundo Pope et al. ${ }^{31}$, os homens tendem a adotar estratégias de mudança corporal diferentes das encontradas em mulheres. Nestas, a prática de dieta (restrição alimentar) e o uso de laxantes e substâncias anorexígenas são mais frequentes, enquanto nos homens são encontrados alta ingesta alimentar, excesso de exercício físico (musculação) e uso de suplementos alimentares e esteroides anabólicos ${ }^{31}$.
No entanto, os dados obtidos nessa pesquisa apontam um número considerável de indivíduos do sexo masculino $(6,43 \%)$ que relatam atitudes alimentares inadequadas. Possivelmente, atitudes até então identificadas apenas em muIheres podem estar em crescimento também na população masculina, o que alerta para uma nova perspectiva de preocupação corporal dos homens, em que não basta apenas um corpo musculoso, mas que também deve apresentar baixo percentual de gordura.

A insatisfação com o peso corporal esteve presente em pequena parcela da amostra, sendo encontrada diferença entre mulheres e homens (17,40\% vs. 2,25\%; $p=0,001)$. Esse resultado é similar a estudo anteriormente realizado na mesma instituição de ensino, em que foi identificada presença de insatisfação corporal em 15,2\% das mulheres e 4,1\% dos homens. No entanto, estudos têm identificado maior frequência de insatisfação em universitárias, variando entre $64,4 \%$ e $87 \%$. Sabidamente, a insatisfação com o peso corporal é mais proeminente em mulheres do que em homens, visto que estes apresentam preocupação acentuada com a muscularidade ${ }^{31}$.

Os comportamentos de checagem corporal estiveram associados às atitudes alimentares inadequadas e à insatisfação corporal em ambos os sexos. Esses dados corroboram a hipótese de a checagem corporal estar associada ao aspecto psicopatológico central dos transtornos alimentares (distúrbios de imagem corporal) ${ }^{6}$ e, ainda, reforçam o fato de que indivíduos com alta frequência desses comportamentos aderem a atitudes alimentares inadequadas ${ }^{6-8,11}$.

Os transtornos alimentares são um desafio atual para os profissionais de saúde, dada a crescente atenção, especialmente das populações ocidentais, ao corpo e à aparência física. Esse desafio é ainda maior para a população masculina, que até pouco tempo atrás era identificada como livre de insatisfação corporal e apresentava baixa prevalência de transtornos alimentares ${ }^{31}$. Embora este estudo tenha apresentado diferenças significativas em relação às mulheres para todas as variáveis, é possível identificar indivíduos do sexo masculino apresentando atitudes alimentares inadequadas e insatisfação corporal, estando essas associadas à checagem corporal. Silva et al. ${ }^{2}$ atentam para a necessidade de estratégias de saúde pública que visem à diminuição da insatisfação com o corpo, tendo em vista os riscos associados a esse acometimento.

Este estudo avança ao apresentar dados de uma variável até então pouco estudada pelos pesquisadores: a checagem corporal. Esta apresentou associação significativa com as atitudes alimentares inadequadas e com a insatisfação com o peso corporal, dados até então desconhecidos na população investigada. No entanto, este estudo apresenta algumas limitações que merecem destaque.

A primeira limitação diz respeito ao $\mathrm{BSQ}$, instrumento desenvolvido por meio de um grupo focal para avaliar a preocupação de mulheres com o seu corpo, e que nesta 
pesquisa foi aplicado também aos homens. Notoriamente, os comportamentos descritos dizem respeito à preocupação com o peso e com a forma corporal, o que recorrentemente está associado à população feminina. No entanto, o BSQ foi adaptado para a população brasileira e apresentou adequadas propriedades psicométricas para ambos os sexos. Para tanto, nesta pesquisa optou-se por utilizar o instrumento como índex da insatisfação com o peso corporal, e não da insatisfação do corpo como um todo. Essa abordagem é utilizada em outros estudos, devido à carência de instrumentos válidos e específicos para a população masculina brasileira.

Ainda com relação aos instrumentos utilizados, o EAT-26, embora tenha apresentado adequada consistência interna em seu processo de adaptação cultural, obteve baixa sensibilidade, reduzido valor preditivo e pobre estabilidade temporal. Segundo Nunes et al. ${ }^{26}$, o instrumento apresenta limitações quanto à sua capacidade de identificar corretamente indivíduos com transtornos alimentares.

O segundo ponto está relacionado ao tipo de estudo conduzido, de corte transversal. Não é possível, por meio dessa abordagem, identificar uma relação causal entre as variáveis. É possível que a checagem corporal seja um efeito tanto da insatisfação corporal quanto das atitudes alimentares inadequadas. Ou seja, o indivíduo insatisfeito e com comer transtornado adota medidas de checagem corporal a fim de avaliar a eficácia no controle sobre o seu corpo. Estudos longitudinais devem ser conduzidos a fim de avaliar o papel de uma variável sobre as outras, clarificando suas interferências. Sabidamente, a orientação sobre a forma de checagem corporal, que enfatiza a análise "positiva" sobre os aspectos da aparência corporal, demonstra melhora da autoestima e da satisfação com o corpo ${ }^{9,10}$.

Por fim, como limitação, tem-se a divisão dos grupos de baixa e alta frequência de comportamentos de checagem corporal. Optou-se por categorizá-los de acordo com as pontuações do questionário, tendo em vista a não existência de normatização para as escalas utilizadas (BCQ e MBCQ). É possível que o baixo número de indivíduos incluídos no grupo de alta frequência decorra dos escores utilizados (frequentemente e muito frequentemente). No entanto, acredita-se que essa metodologia seja adequada a fim de incluir nesse grupo apenas os indivíduos com alto padrão de checagem corporal, diminuindo, assim, a possibilidade de inclusão de indivíduos que apresentassem escores próximos aos valores obtidos pelo grupo, por exemplo, na adoção da média ou mediana do grupo para tal finalidade.

\section{CONCLUSÃO}

Os dados desta pesquisa permitem concluir que o comportamento de checagem corporal está presente em jovens universitários de ambos os sexos e que ele está associado a dois fatores de risco para o desenvolvimento de transtornos alimentares: a insatisfação corporal e as atitudes alimentares inadequadas. Indivíduos do sexo feminino apresentaram maior insatisfação corporal, checagem corporal e atitudes alimentares inadequadas do que os indivíduos do sexo masculino. O conhecimento da associação dessas variáveis e das idiossincrasias de cada sexo é de suma importância para o desenvolvimento de estratégias de saúde pública e de orientação para a população jovem adulta.

\section{CONTRIBUIÇÕES INDIVIDUAIS}

Pedro Henrique Berbert de Carvalho e Maria Elisa Caputo Ferreira - Participaram da concepção e do desenho do estudo, da análise e da interpretação dos dados, da redação e da revisão do conteúdo intelectual e aprovaram a versão final a ser publicada.

Juliana Fernandes Filgueiras, Clara Mockdece Neves e Fernanda Dias Coelho - Participaram da análise e da interpretação dos dados, da redação e da revisão do conteúdo intelectual e aprovaram a versão final a ser publicada.

\section{CONFLITO DE INTERESSES}

Os autores declararam não haver conflitos de interesse.

\section{AGRADECIMENTOS}

A todos os participantes, pela disponibilidade e incentivo para que os alunos participassem desta pesquisa; ao Laboratório de Estudos do Corpo (Labesc), pela disponibilização dos instrumentais do estudo; ao Conselho Nacional de Desenvolvimento Científico e Tecnológico (CNPq), pela bolsa de estudos conferida ao primeiro autor deste manuscrito.

\section{REFERÊNCIAS}

1. American Psychiatric Association. Diagnostic and statistical manual of mental disorders. $4^{\text {a }}$ ed. Washington: American Psychiatric Association; 1994.

2. Silva DAS, Nahas MV, de Sousa TF, Del-Duca GF, Peres KG. Prevalence and associated factors with body image dissatisfaction among adults in southern Brazil: a population-based study. Body Image. 2011;8(4):427-31.

3. Philippi ST, Latterzza AR, Cruz ATR, Ribeiro LC. Pirâmide alimentar adaptada: guia para escolha dos alimentos. Rev Nutr. 1999;12(1):65-80.

4. Scherer FC, Martins CR, Pelegrini A, Matheus SC, Petroski EL. Imagem corporal em adolescentes: associação com a maturação sexual e sintomas de transtornos alimentares. J Bras Psiquiatr. 2010;59(3):198-202.

5. Alvarenga MS, Scagliusi FB, Philippi ST. Comportamento de risco para transtorno alimentar em universitárias brasileiras. Rev Psiq Clín. 2011;38(1):3-7.

6. Shafran R, Fairburn CG, Robinson P, Lask B. Body checking and its avoidance in eating disorders. Int J Eat Disord. 2004;35(1):93-101. 
7. Fairburn CG, Shafran R, Cooper Z. A cognitive behavioral theory of anorexia nervosa. Behav Res Ther. 1999;37(1):1-13.

8. Mountford V, Haase A, Waller $\mathrm{G}$. Is body checking in the eating disorders more closely related to diagnosis or to symptoms presentation? Behav Res Ther. 2007;45(11):2704-1.

9. Delinsky SS, Wilson GT. Mirror exposure for the treatment of body image disturbance. Int J Eat Disord. 2006;39(2):108-16.

10. Veale D, Riley S. Mirror, mirror on the wall, who is the ugliest of the mall? The psychopathology of mirror gazing in body dysmorphic disorder. Behav Res Ther. 2001;39(12):1.381-93.

11. Walker DC, Anderson DA, Hildebrandt T. Body checking behaviors in men. Body Image. 2009;6(3):164-70.

12. Alfano L, Hildebrandt T, Bannon K, Walker C, Walton KE. The impact of gender on the assessment of body checking behavior. Body Image. 2011;8(1):20-5.

13. Petribú MMV, Cabral PC, Arruda IKG. Estado nutricional, consumo alimentar e risco cardiovascular: um estudo em universitários. Rev Nutr. 2009;22(6):837-46.

14. Miranda VPN, Filgueiras JF, Neves CM, Teixeira PC, Ferreira MEC. Insatisfação corporal em universitários de diferentes áreas de conhecimento. J Bras Psiquiatr. 2012;61(1):25-32.

15. Alvarenga MS, Philippi ST, Lourenço BH, Sato PM, Scagliusi FB. Insatisfação com a imagem corporal em universitárias brasileiras. J Bras Psiquiatr. 2010;59(1):44-51.

16. Bosi MLM, Uchimura KY, Luiz RR. Eating behavior and body image among psychology students. J Bras Psiquiatr. 2009;58(3):150-5.

17. Thomas JR, Nelson JK, Silverman SJ. Métodos de pesquisa em atividade física. São Paulo: Artmed; 2007.

18. Reas DL, Whisenhunt BL, Netemeyer R, Williamson DA. Development of the Body Checking Questionnaire: A self-report measure of body checking behavior. Int J Eat Disord. 2002;31(3):324-33.

19. Campana ANNB. Tradução, adaptação transcultural e validação do "Body Image Avoidance Questionnaire (BIAQ)" e do "Body Checking Questionnaire $(B C Q)$ " para a língua portuguesa no Brasil [mestrado]. Campinas: Universidade Estadual de Campinas; 2007.
20. Hildebrandt T, Walker DC, Alfano L, Delinsky C, Bannon K. Development and validation of a Male Specific Body Checking Questionnaire. Int J Eat Disord. 2010;43(1):77-87.

21. Carvalho PHB, Conti MA, Cordás TA, Ferreira MEC. Tradução para o português (Brasil), equivalência semântica e consistência interna do Male Body Checking Questionnaire (MBCQ). Rev Psiq Clín. 2012;39(2):74-5.

22. Carvalho PHB, Conti MA, Ribeiro MS, Amaral ACS, Ferreira MEC. Avaliação psicométrica do Male Body Checking Questionnaire. Psicologia: Reflexão e Crítica (no prelo).

23. Cooper P, Taylor MJ, Cooper Z, Fairburn CG. The development and validation of the Body Shape Questionnaire. Int J Eat Disord. 1987;6(4):485-94.

24. Di Pietro M, Silveira DX. Internal validity, dimensionality and performance of the Body Shape Questionnaire in a group of Brazilian college students. Rev Bras Psiquiatr. 2009;31(1):21-4.

25. Garner DM, Olmsted MP, Bohr Y, Garfinkel PE. The eating attitudes test: Psychometric features and clinical correlates. Psychol Med. 1982;12(4):871-8.

26. Nunes MA, Camey S, Olinto MTA, Mari JJ. The validity and 4-year test-retest reliability of the Brazilian version of the Eating Attitudes Test-26. Braz J Med Biol Res. 2005;38(11):1655-62.

27. Avila-Funes JA, Gutierrez-Robledo LM, Ponce-de-Leon-Rosales S. Validity of height and weight self-report in Mexican adults: results from the national health and aging study. J Nutr Health Aging. 2004;8:355-61.

28. Kawada T, Suzuki S. Validation study on self-reported height, weight, and blood pressure. Percept Mot Skills. 2005;101:187-91.

29. World Health Organization. WHO Expert Committee on Physical Status: The use and interpretation of anthropometry physical status. Geneva: World Health Organization; 1995 (WHO Technical Report Series, v. 854).

30. Nunes MA, Barros FC, Olinto MTA, Camey S, Mari JDJ. Prevalence of abnormal eating behaviors and inappropriate methods for weight control in young women from Brazil: a population - based study. Eat Weight Disord. 2003;8:100-6.

31. Pope H, Phillips KA, Olivardia R. The Adonis complex: the secret crisis of male body obsession. New York: The Guilford Press; 2000. 